\title{
Photometry with adaptive optics: A first guide to expected performance $^{\star}$
}

\author{
O. Esslinger and M.G. Edmunds \\ Dept. of Physics and Astronomy, University of Wales, Cardiff, CF2 3YB, UK \\ e-mails: O.Esslinger@astro.cf.ac.uk, M.Edmunds@astro.cf.ac.uk
}

Received March 21; accepted November 12, 1997

\begin{abstract}
This paper aims to help prospective observers estimate the likely performance of adaptive optics for photometric observations. Both real and simulated adaptive optics data are used in specific examples covering the major observational situations. These illustrate the different sources of uncertainty: seeing fluctuations, presence of both a large halo and residual fluctuations in the point spread function, and angular anisoplanatism. The astronomical cases studied include isolated sources, faint structures around a star, and crowded stellar fields. The photometric performance image deconvolution is also examined. No attempt is made to investigate exhaustively all possible atmospheric conditions and observing configurations, but the discussion should be a useful guide to the feasibility of using adaptive optics in astronomical programmes requiring particular photometric accuracies.
\end{abstract}

Key words: turbulence - atmospheric effects instrumentation: miscellaneous - method: data analysis - techniques: photometric — stars: individual: Betelgeuse

\section{Introduction}

Although several papers have dealt with the image quality of an adaptive optics system (see e.g. Wilson \& Jenkins (1996) for theoretical performance and Tessier (1997) for real results), none has focused specifically on the quality of photometry possible with these images. Since the accuracy of photometry is often crucial in the astrophysical conclusions drawn from observations, we attempt to give a guide for the average observer of the performance and limitations that can be expected when an adaptive optics system is used. Most of the examples given here

\footnotetext{
Send offprint requests to: M.G. Edmunds

* Based on observations obtained at the European Southern Observatory, La Silla, Chile.
}

use Strehl ratios between 0.15 and 0.3 , which is typical of fair correction currently achievable in $H$ and $K$ wavelength bands, and have been obtained with the ADONIS system at the 3.6-metre ESO telescope. We do not try to estimate performances in a complete set of atmospheric conditions, since such a complete error analysis would really only be appropriate to a particular adaptive optics system, and our intention is to provide "rule-of-thumb" estimates rather than investigating the ultimate accuracy achievable under special circumstances. Table 6 at the end of this paper gives a brief summary of typical achievable accuracies for near-infrared photometry in a range of astronomical programmes.

Using adaptive optics introduces problems which are not usually encountered in normal photometry: global variations of the PSF with time, fluctuations in the large halo surrounding the core of the PSF, the presence of features in the PSF due to residual aberrations and variations of the PSF with the position in the image (due to the dependence of phase perturbations on position in the sky, called angular anisoplanatism). The influence of these problems on photometric performance is considered in several cases: uncrowded fields, faint structures around unresolved bright objects, and crowded fields. These examples should give a useful guide to performance in most astronomical photometric programmes that might be tackled using adaptive optics. We also study the effect on photometry of image deconvolution. This article looks at errors directly linked to the use of adaptive optics, and therefore most of the time ignores issues such as the presence of noise (sky background, photon or readout noise) or other problems with which the astronomer is used to dealing (for instance, flat fielding).

Section 2 introduces photometry with arrays and reviews the particular problems introduced by the use of an adaptive optics system. It also describes the observational data which is a vital input to our estimates. In Sect. 3, the potential advantages of using adaptive optics for photometry are illustrated by two examples. Section 4 then studies the impact of the limitations of adaptive optics in 
the simple case of an uncrowded field. Section 5 assesses their influence in the more complex case of a crowded field. This includes the detection of a faint companion or complex structure around a bright object, and the case of a field containing a large number of objects. The effects of deconvolution on photometry are considered in Sect. 6 . Conclusions are drawn in Sect. 7, including a brief discussion of the implications of the use of laser guide stars, and simple advice on estimating photometric errors.

\section{Photometry with arrays}

There are currently two different methods of performing photometry with arrays: aperture photometry, for uncrowded fields, and point spread function fitting, for crowded fields. Aperture photometry is the equivalent of classical diaphragm photometry applied to an array frame: a given aperture is chosen and the light of the object studied is integrated on this aperture. The sky background is estimated by a measurement in an annulus around the object of interest. This area must be close enough to the star so that its background and the aperture's are the same, but not too close in order to minimise the contribution of the object in the annulus. When dealing with crowded fields, aperture photometry is no longer applicable as several sources can contribute together to the intensity in an aperture. In this case, another method has to be used: point spread function fitting. This consists of fitting a model of the stellar images to the data via a leastsquares algorithm, allowing the study of several stars at the same time.

Both photometric methods rely on some strong assumptions. Basically, the point spread function has to be known very accurately and it should be smooth, stable with time and constant over the whole field of view. This is approximately the case in normal ground-based observations. The PSF is then seeing-limited, most of the energy is contained within a circle of diameter 1 arcsec or so, and the profile is fairly smooth and constant with time and position. But the requirements are no longer met when an adaptive optics system is used. As we will soon see, the global shape of the PSF then varies a lot with time and also with the position of the object on the field of view. Furthermore, the PSF is surrounded by a halo which is affected by strong fluctuations as well. Last, the PSF is not smooth but shows irregularities due to residual aberrations. All these factors mean that performing photometry with adaptive optics is much less straightforward than with a seeing limited system - although the use of this technique can still bring considerable improvement.

\subsection{Variations with time}

The first drawback of adaptive optics as far as photometry is concerned is the variability of the PSF with time. The variations of the PSF with time have two causes.
First, the shape of the PSF and its Strehl ratio depend strongly on the seeing, which is known to vary rapidly with time. The variations of the seeing therefore imply strong fluctuations of the PSF with time. Secondly, some PSF variations are also due to wavefront sensor noise and to fluctuations in the high-order spatial modes of the wavefront that are not corrected by the adaptive optics system. These fluctuations would be present even if the seeing were constant. Tessier (1995) showed that the first possibility, seeing fluctuations, is probably the predominant source of PSF variations, even if it has not yet been proved decisively. Whatever the reason, the PSF cannot be considered stable with time, which poses a major problem for both photometric methods.

The usual procedure in aperture photometry is to observe both the objects of interest and photometric standards for calibration purposes. The science objects and these standards are unlikely to be very close in the sky, which means that they have to be observed at different times. The time variations described above therefore mean that the PSF changes slightly between the images of the targets and the standards. In the case of point spread function fitting, the problem is more obvious as the method requires an accurate knowledge of the point spread function, even when only relative photometry is performed. But in most applications of adaptive optics in crowded fields, search for faint companions around a bright object or stellar clusters, no independent PSF can be found in the science frames, either because only the bright object itself is available or because the field is too crowded to yield an isolated star. This means again that a calibration star has to be observed at a different time and therefore in slightly different conditions, hence the problem. Note that a possible way to overcome the problem of global PSF variations could be to reconstruct the PSF using control loop data, as proposed by Veran et al. (1997)

We have only considered global variations of the PSF linked to Strehl ratio variations so far. Another problem arises when the purpose of an observation is to detect faint structures around a star. In the common case where the adaptive optics correction is not perfect (i.e. the partial correction regime), the PSF is in fact the sum of two components: a central core which approximately corresponds to the diffraction-limited Airy profile and a large halo surrounding this core and due to the uncorrected or partially corrected high-order modes. The presence of this halo is going to be a major drawback for the detection of faint structures around a star. First, given its origin, the halo strongly fluctuates with time. This means that from one frame of an object to another, the faint structures in the PSF are different, a tendency which is enhanced by the unavoidable presence of noise in the image and in the wavefront sensor measurements. Secondly, even if this halo were stable with time, it would still depend on parameters such as the brightness of the object, its shape, its colour or its position in the sky. Changes in the halo can 
therefore appear in the usual case where several stars have to be compared. This second type of variation can be reduced by a careful choice of the calibration stars and by applying the procedure presented for example in Tessier (1995) (calibration star near the astronomical object, with the same flux, long integration time, short delay between observations). But the first type will always be present. Longer integration times can reduce the variations but never completely eliminate them. This is a serious problem when trying to detect structures such as a companion or an extended source around a star, but obviously also affects photometric measurements of such faint structures. We can add to this problem the fact that, for any natural guide star system, a bright star is needed if the science object is too faint to be used itself. This bright star is going to be surrounded by a halo whose fluctuations might dominate the faint science object and prevent any accurate photometric measurement.

\subsection{Other sources of error}

A third problem is the presence of residual features in the PSF: spikes due to the secondary mirror supports, lumps in the diffraction ring due to some imperfectly corrected mode (for instance the coma) and also faint artifacts all around the core due to fixed residual aberrations in the adaptive optics system. All these features are affected by global variations and halo fluctuations with time, and by photon noise. They therefore vary with time in a way that cannot be predicted, and introduce a new source of difficulties in the detection of a faint objects and their photometry.

The last major problem of adaptive optics is angular anisoplanatism. The phase deformations induced by the atmosphere in two different directions are not the same. As a consequence, the PSF varies with the position in the field of view. This is a serious problem for aperture photometry in moderately crowded fields and for point spread function fitting as both methods rely on a constant shape for the PSF. In the case of aperture photometry, the fraction of light contained in a given aperture will depend on the position of an object. For point spread function fitting, slicing the field of view into subimages can help, but it requires determination of a PSF for each area and also introduces some calibration problems between different subimages.

We have only considered performing photometry out of direct images so far. Thanks to a very sharp core in the PSF, adaptive optics images can directly yield very good results. Nevertheless their full exploitation requires the use of efficient deconvolution methods such as the maximum entropy method (Gull \& Daniell 1978) or the Lucy-Richardson algorithm (Lucy 1974; Richardson 1972) The use of these methods introduces a new kind of error. Deconvolution methods usually yield images that are in qualitative agreement with the true images. But this is not enough because a quantitative agreement is required to perform photometry. We therefore have to check if deconvolved images can be used to obtain quantitative data or if the deconvolution algorithms introduce large errors in the brightness of the different objects. Such a problem is not new and can be compared to that faced by users of the HST before correction of spherical aberration (see e.g. White \& Burrows 1990). The problem is even more serious with adaptive optics because the PSF is usually not known precisely. Thus, another source of error has to be taken into account: the influence of a badly determined PSF on the photometric accuracy of deconvolved images.

To conclude this section, we can briefly summarise the problems of photometry with adaptive optics:

1. Global PSF variations between the science objects and the photometric standards or calibration stars.

2. Fluctuations in the faint halo surrounding the core of the PSF.

3. Presence of residual features in the PSF.

4. PSF variations on the field of view due to angular anisoplanatism.

5. Biases introduced by the use of deconvolution methods.

6. Deconvolution using a badly determined PSF.

This study is devoted to the errors directly linked to the use of adaptive optics. We will not take into account other sources such as the precision of the flat fields or colour transformations. In most cases, we will also assume that no noise (photon noise or sky background) is present in the images.

\subsection{Origin of our data}

Two kinds of data have been used in this work: actual images and simulated point spread functions. The actual images were obtained with the COME-ONPLUS/ADONIS system installed at the 3.6-metre telescope of the European Southern Observatory in La Silla (Beuzit et al. 1994). Images acquired during different runs in 1993, 1995 and 1996 were used. These included observations of Betelgeuse (Esslinger 1997) and of several massive stars (see e.g. Heydari-Malayeri et al. 1997a,b). The observations of Betelgeuse were carried out with a narrow band filter at $2.08 \mu \mathrm{m}$. The others were performed in the $H$ and $K$ broad bands, and a few in the $J$ band. The science objects were bright enough to be used as their own wavefront correction reference.

To illustrate the theoretical benefits of using adaptive optics and to assess the consequences of angular anisoplanatism, we used simulated point spread functions constructed using the method described in Wilson \& Jenkins (1995) and kindly provided by the authors. The atmospheric model assumed two thin turbulent layers, one at an altitude of 1 kilometres and another at 5 kilometres. The lowest layer was twice the strength of the upper one, 
which is believed to be a reasonable model for the atmosphere above another good astronomical site, La Palma. Note that these simulations assumed no central obscuration of the telescope and a correction of 20 modes (this was a computation time limitation).

\section{The benefits of adaptive optics}

Before dealing with the consequences of all the problems presented above, we first present the benefits of using adaptive optics in two illustrative examples. The theoretical advantages of using an adaptive optics system in order to perform photometry are clear. For isolated objects, the increase in Strehl ratio means a much better contrast against the background noise, and the higher concentration of flux allows a reduction in the size of the aperture used for measurement, which means a significant reduction of noise. For crowded fields, the use of adaptive optics has a further advantage: by sharpening the image, it reduces confusion and separates close sources which could otherwise have been considered as single objects by the point spread function fitting algorithm. In this section, we give an illustration of the theoretical benefits of adaptive optics in two such cases, a single star observed against the sky background and a small star cluster, without taking into account any of the sources of noise described before. We use the simulated PSF constructed using the method described in Wilson \& Jenkins (1995) and kindly provided by the authors. The PSFs are obtained on-axis for three values of $D / r_{0}: 10,7.5$ and 5 , corresponding approximately to the $J, H$ and $K$ bands on a 4-meter telescope in good seeing conditions $(0.75$ arcsec seeing at $0.5 \mu \mathrm{m})$.

\subsection{Isolated objects}

We first simulated a single star observed against the sky background in the three bands $J, H$ and $K$. In each band, we considered three cases: an uncorrected image, an image obtained with only tip/tilt correction, and an image with an adaptive optics correction of 20 modes. The value of the noise in each band was chosen to yield a signal to noise ratio of 2 for the peak for the uncorrected image. The IRAF/NOAO APPHOT aperture photometry package was then applied to each image in order to work out the flux contained in a given aperture. The radius of this aperture was chosen to be 1.22 times the Full Width at Half Maximum of each image. In the diffraction-limited case, this corresponds to $1.22 \lambda / D$, i.e. the radius of the first dark ring. This choice of aperture is not optimised but simply serves as an illustrative example.

For each band and each level of correction, we created a set of 10 images with a different statistical realisation of the background noise. We computed the flux of the star in the chosen aperture for each image, and finally worked out the rms variations of the corresponding magnitudes, which gave us an estimate of the error on the magnitude.
Table 1. Error in the magnitude measured through aperture photometry for different bands and different level of correction. The noise was gaussian and its rms level set in each band to yield a signal to noise of 2 for the peak of the uncorrected PSF

\begin{tabular}{clll}
\hline Correction & \multicolumn{1}{c}{$J$} & \multicolumn{1}{c}{$H$} & \multicolumn{1}{c}{$K$} \\
\hline No correction & 0.05 & 0.05 & 0.05 \\
Tip/tilt & 0.015 & 0.010 & 0.010 \\
20 modes & 0.002 & 0.003 & 0.005 \\
\hline
\end{tabular}

The results are presented in Table 1, which gives the uncertainty on the magnitude in each band and for each level of correction. In the case of no applied correction, the uncertainty is approximately the same whatever the band because we set the same signal to noise for each uncorrected image. When a tip/tilt correction is applied, the signal to noise ratio of the peak is multiplied by 2.6 in $J$, 3.7 in $H$ and 5.3 in $K$. This leads to a decrease in the uncertainty on the magnitude by a factor 2 or so in $J$ and about 3 in $H$ and $K$. When a correction of 20 modes is applied, the signal to noise is multiplied by 40 in $J, 33$ in $H$ and 40 in $K$ (compared to the uncorrected case). The uncertainty of the magnitude is then divided by 25 in $J$, 17 in $H$ and 10 in $K$, the relative improvement being better at shorter wavelength because the increase in signal to noise is greater. This example clearly shows that the use of adaptive optics on isolated faint objects theoretically leads to a huge improvement in the accuracy of photometry, by at least a factor 10, thanks to a better contrast and a higher concentration of the flux.

\subsection{Crowded fields}

In order to illustrate the advantages of adaptive optics in the very usual case of a crowded field, for instance a star cluster, we created artificial images of a cluster containing 15 objects: one bright star whose magnitude was taken as a reference, 2 stars 1.25 magnitudes fainter, 4 stars 2.5 magnitudes fainter and 8 star 3.75 magnitudes fainter. One image was created with an uncorrected $H$ band PSF (Strehl 0.016), one with a tip/tilt-corrected $H$-band PSF (Strehl 0.061) and one with a corrected $H$ band PSF (20 modes, Strehl 0.55). The 15 stars were scattered on a area of $3 \times 3 \operatorname{arcsec}^{2}$, which yielded a density of about 1.7 stars per $\operatorname{arcsec}^{2}$. Some gaussian noise was added on the three images with a level chosen to be 5 magnitudes fainter than the peak of the brightest star in the uncorrected case. Figure 1 presents the three images, uncorrected on the left, tip/tilt corrected in the middle and and fully corrected on the right. In the corrected images, all the components are clearly visible, whereas in the uncorrected one, the faintest components can only be 
identified after a subtraction of the brightest stars. This clearly has consequences for the precision of photometry.

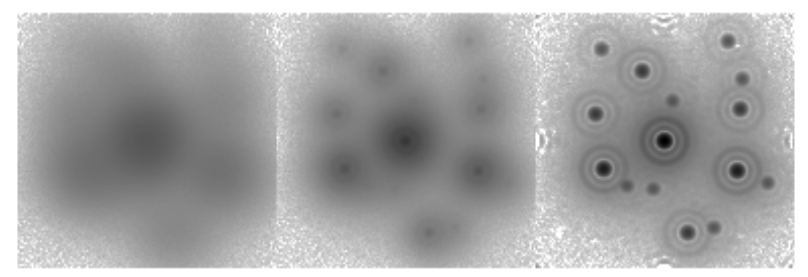

Fig. 1. Uncorrected, tip/tilt corrected and fully corrected images of a star cluster created with simulated PSFs in the $H$-band were used. A logarithmic scale is used to show the faintest stars. The use of adaptive optics clearly brings a huge improvement, both for detection of objects and for photometry

To assess the improvement in photometric accuracy in this case, we applied the IRAF/NOAO DAOPHOT point spread function fitting package to the three images to work out the magnitude of each component of the cluster. The PSF was supposed to be perfectly known. Comparing the results to the actual magnitudes in the original artificial image, we were able to work out the error on each measurement. We then computed the rms error for each group of star with a given flux in order to obtain a final value: the average uncertainty on the flux of a star with a given difference in magnitude relative to the brightest object of the field. The results are presented in Table 2 . In this case again, the use of adaptive optics leads to a huge increase in precision. For the three differences in magnitude considered, the improvement in precision between the uncorrected and the fully corrected images varies between 15 and 35 and very accurate results can theoretically be obtained. For instance, for a difference in magnitude of 3.75 , the uncertainty in the uncorrected image makes the measured values virtually useless, whereas the corrected image yields very useful results with a precision better than 5 per cent. Here again, thanks to the increase in contrast and the better concentration of energy, adaptive optics theoretically leads to much more accurate photometric results.

The problem is that the two situations in this section have been simulated without taking into account the sources of noise introduced in Sect. 2, and these results must therefore be considered optimistic. The rest of this article is an assessment of the influence of each source of noise on photometric accuracy, and it will show that the improvements illustrated above cannot be fully achieved in reality.
Table 2. Error in the magnitudes of the cluster's components obtained by a point spread function fitting algorithm. Three differences in magnitude relative to the brightest object and three levels of correction are considered. The results are obtained with simulated PSFs in the $H$ band

\begin{tabular}{clll}
\hline Correction & $\Delta m=1.25$ & $\Delta m=2.50$ & $\Delta m=3.75$ \\
\hline No correction & 0.07 & 0.08 & 0.65 \\
Tip/tilt & 0.005 & 0.025 & 0.07 \\
20 modes & 0.002 & 0.003 & 0.04 \\
\hline
\end{tabular}

\section{Uncrowded fields and aperture photometry}

\subsection{Variations of the PSF with time}

Aperture photometry involves measuring the amount of light contained in a given aperture for each object. The same procedure is usually applied on the science objects and on a few photometric standards. In most cases, the science objects and the standards have to be observed at different times. This introduces two problems. Because of the constantly changing PSF of an adaptive optics system, the fraction of light contained in a given aperture also varies, which introduces an error in the calibration procedure. Note that this is a drawback because in order for adaptive optics to produce a gain, the size of the aperture has to be reduced. The second problem is also related to PSF variations, but this time because of a different correction for the science objects and the photometric standards. The difference is essentially created by a different level of noise in the wavefront sensor and can be minimised by carefully selecting the brightness of the standard stars and their spectral type, even if some slight differences caused by their colour or brightness might remain. Tessier (1995) showed that when the different objects are carefully matched, the main source of variations in the PSF is seeing fluctuations. In our assessment of the accuracy of aperture photometry, we therefore assumed that the second source of error was negligible compared to the first one - but note that this does require careful matching.

In order to estimate the errors induced by PSF variations on aperture photometry, we analysed several sets of data of Betelgeuse and massive stars using the IRAF/NOAO APPHOT package. We considered sets of 4 or 5 successive frames of single stars taken in the $H$ and $K$ bands or in a narrow band filter at $2.08 \mu \mathrm{m}$ during these runs. The individual observations had integration time varying from 1 to 90 seconds. For each set of frames, we worked out the rms variation of the magnitude measured in a given aperture. To define the sizes of these apertures, we decided to use areas defined by the three first dark rings of the unobstructed diffraction limit of the 
telescope (even if the images were not actually diffractionlimited). The corresponding sizes were given by Born \& Wolf (1970):

$$
1.2 \frac{\lambda}{D}, 2.2 \frac{\lambda}{D} \text { and } 3.2 \frac{\lambda}{D}
$$

In $K$ for example, this corresponded to aperture diameters of $0.31,0.56$ and 0.82 arcsecs.

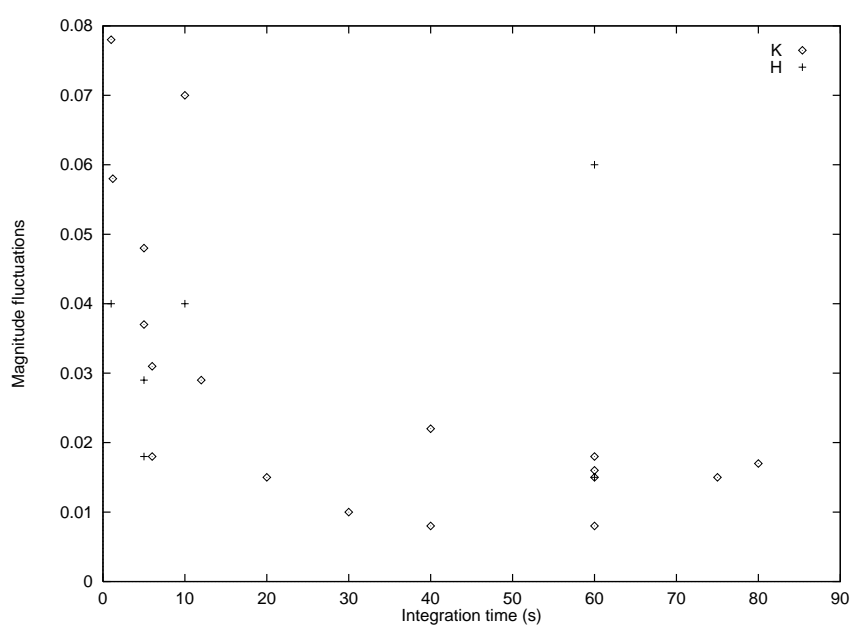

Fig. 2. Rms fluctuations of the magnitude measured in a given aperture as a function of the integration time of a single frame. The size of the aperture is defined by the second dark ring of the diffraction limit of the telescope. Crosses are data taken in the $H$ band and diamonds data taken in the $K$ band. The data were obtained during four different runs with ADONIS

Figure 2 presents the results in the case of an aperture defined by the second dark ring. The rms variation of the magnitude measured in an aperture is given as a function of the integration time for one frame. The pixel size was either 35 or 50 milliarcsec and the four observing runs gave results consistent with each other (for example, for an integration time of 1 minute, different data from the four runs all yielded fluctuations of 0.015 magnitude or so). The signal to noise ratios of the images (defined by the peak of the PSF and the background noise throughout this article) varied between 300 and 45000 , with most of them above 7000 . In $H$, the Strehl ratios were between 0.05 and 0.15 , and in $K$, between 0.15 and 0.35 . Each rms fluctuation of the flux was computed from measurements on 4 or 5 successive images. We verified in each case that the error due to photon or background noise was clearly smaller than the total error (it always amounted to less than 0.01 magnitude).

Figure 2 shows that globally the rms variations behave like $1 / \sqrt{T}$, where $T$ is the integration time. Such a behaviour can be expected if the fluctuations of the magnitude are completely uncorrelated from one frame to another. The data globally follow this trend even if the spread is quite large and some points stand out. The spread is not surprising as parameters other than the integration time (such as the seeing fluctuations or the brightness of the guide star) are also important.

Figure 2 gives an estimate of the precision of aperture photometry when the science objects and the photometric standards have to be observed at different times. Note that the results apply only to near-infrared observations, as they depend on the rate of change of the Strehl upon the coherence length of the atmosphere, and therefore on the wavelength. For exposure times between 20 and 90 seconds, seeing fluctuations impose a limit to the accuracy of photometry at a level of about 0.01 or 0.02 magnitudes. For exposure times of a few seconds, this limit increases to 0.04 magnitude and more. We also tried to work out the magnitude variations for longer exposure times. These attempts showed that the variations tend to get larger than 0.02 magnitudes rather than smaller. This can be explained by the fact that for long exposure times, the amount of light in an aperture is determined by the slow variation in the atmosphere turbulence and the successive magnitude estimates are no longer uncorrelated. The fluctuations are then controlled by the amplitude of the slow variations of the seeing and increase with the exposure time. A first conclusion that can be drawn from Fig. 2 is the fact that aperture photometry measurements with adaptive optics are unlikely to yield results more precise than 0.01 or 0.02 magnitude. The theoretical accuracies obtained in the previous section for a 20 -mode correction are, for instance, far too optimistic. Nevertheless, this limitation still allows much better precision than in the seeinglimited case.

\subsection{Influence of the aperture size}

An important parameter which determines the amplitude of the fluctuations is the size of the chosen aperture. The larger the aperture, the smaller the variations. In order to estimate the influence of the aperture size, we analysed a set of 8 frames from our observations of Betelgeuse. The integration time was 6 seconds and the observations were spread over a little more than 1 hour. The signal to noise ratios varied between 25000 and 30000 , with one exception at 17000 . The Strehl ratios were between 0.20 and 0.26 , with the same exception at only 0.14 , and the average value was 0.22 . In each frame we measured the Strehl ratio and the flux contained in three different apertures, defined as before by the three first dark rings.

Figure 3 presents the result. The magnitude computed in the three different apertures are given as a function of the Strehl ratio in the frame. Magnitudes are given relative to the average magnitude for each aperture and Strehl ratios relative to the average Strehl ratio. Figure 3 gives variations in flux induced by fluctuations in Strehl ratio. Parameters like the exposure time or the brightness of the object do not influence these variations. Figure 3 is 


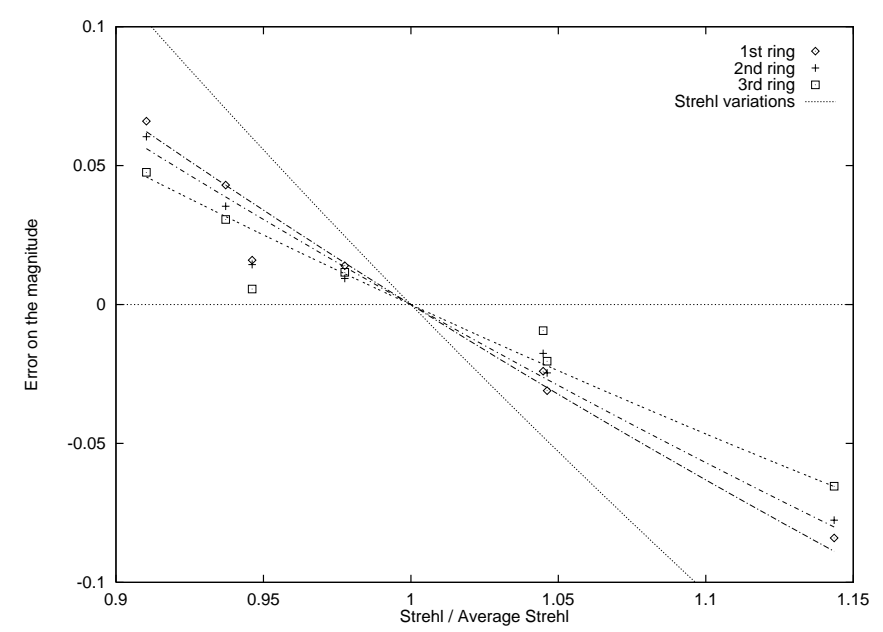

Fig. 3. Variation in magnitude as a function of the variation in Strehl ratio. All values are given relative to the averages on the whole set of data. Three different aperture are show: one defined by the first dark ring (diamonds), the second dark ring (crosses) and the third one (squares). The data were taken in the $K$ band with an exposure time of 6 seconds and a pixel size of 0.035 arcsec. The average Strehl was 0.22

therefore very general and will be the same for any other observation (at least for a similar range of Strehl ratios and a similar PSF shape). The line denoted Strehl variations represents a case where the variation in flux is exactly equal to the variation in Strehl ratio. Its equation is:

$m-\bar{m}=-2.5 \log (S / \bar{S})$.

Figure 3 shows that the actual variations are smaller, which could be expected as the use of a large aperture attenuates the fluctuations. It also shows that the variation in flux for an aperture is approximately proportional to the variation in Strehl. We could plot three lines which approximately fitted the data for the three different apertures. Each line has the following equation:

$m-\bar{m}=-\kappa \times 2.5 \log (S / \bar{S})$.

where $\kappa$ is a factor depending on the size of the aperture. The values of $\kappa$ that best fit the data are 0.61 for the first dark ring, 0.55 for the second and 0.45 for the third, with an uncertainty of about 0.02 . Note that another frame with a poor Strehl ratio $(S / \bar{S}$ about 0.64 , not shown on the figure) also fitted these lines very well.

Figure 3 gives a way of estimating the influence of Strehl ratio or seeing fluctuations on the variations of the magnitude measured in an aperture. It therefore makes a correction possible from one frame to another. But given that some data do not fit the three lines well, an uncertainty remains in this procedure, of about 0.02 magnitude in this particular case. Moreover, to correct for the Strehl ratio effect, a way to measure this parameter is needed: the Strehl ratio cannot be measured in the usual way from the images, because this method requires an absolute measurement of the total flux, which we do not have. From the estimates of the factor $\kappa$, we can also assess the improvement brought by using a larger aperture. First the use of an aperture defined by the first dark ring decreases the fluctuations of the magnitude of 40 per cent compared to pure Strehl variations. When going from the first to the second dark ring, magnitude variations still decrease by 10 per cent or so. When going to the third dark ring, the variations still decrease by about 25 per cent.

Finally, we also tried to apply point spread function fitting. Of course, this method normally applies to crowded fields, but it was nevertheless interesting to assess its behaviour. The results showed that the magnitude variations could also be approximately described by Eq. (3), even though the fit was less accurate than before. The coefficient $\kappa$ was found to be 0.77 or so, well above the values for aperture photometry, vitiating the use of the method in the case of a single source in an uncrowded field.

\subsection{Improvement from a real-time selection}

One possible way to reduce the fluctuations between different frames would be to perform a real-time selection during the observations. For example, during each exposure, the Strehl ratio or another parameter could be checked at a very fast rate, say every hundredth of a second, and a fast shutter would close the camera when this parameter is beyond a threshold value. Different selection criteria could be applied. For example, only the Strehl ratios above a given level can be taken into account. Or, in order to favour consistency, only the ones near an average value could be chosen. Obviously, such a procedure should only be applied to bright objects, when seeing fluctuations dominate the effects of photon or read-out noise.

We assessed these two possibilities using a set of 300 20-ms exposures of Betelgeuse. We considered 5 sets of 60 frames in order to simulate 5 successive 1.2 -second frames. We then applied a selection criterion to the frames. In one case, we rejected all the frames with a Strehl ratio below a certain level, in the other one, we rejected all the frames with a Strehl ratio too far from the average value (on the 300 frames). We could then compute an average image for each $1.2 \mathrm{~s}$-exposure frame and work out the rms variation of the magnitude measured in these 5 frames. We considered 3 different levels of selection: rejection of 50,75 or 150 frames, corresponding to one frame out of 6 , one out of 4 or half the frames. The results are presented in Table 3.

Table 3 shows that such a selection can be quite effective in reducing magnitude fluctuations. For example, keeping the shutter open during only half the exposure time reduces the magnitude fluctuations by about 60 per cent. In our simulations, we kept a constant integration time without taking into account the time during which the shutter was actually open, which leads to a variable 
Table 3. Rms variations of the magnitude from frame to frame when selection is applied, relative to the variation in the case without selection (which corresponds to 0.06 magnitude)

\begin{tabular}{lcc}
\hline Selection & Best Strehl sel. & Average Strehl sel. \\
\hline 1 out of 6 & 0.038 & 0.041 \\
1 out of 4 & 0.033 & 0.036 \\
1 out of 2 & 0.025 & 0.023 \\
\hline
\end{tabular}

effective integration time. For instance, when we rejected an average of half the frames, the number of frames actually used in each 1.2-second exposure varied between 12 and 47 (instead of the theoretical average value of 30). A better way to perform the selection would be to consider a variable integration time, dependent on the variations of the Strehl ratio, in order to obtain a fixed effective integration time. This would not greatly affect the level of magnitude fluctuation and yield a coherent set of images with the same effective integration times and signal to noise ratios.

\subsection{Angular anisoplanatism}

The second problem as far as aperture photometry is concerned is angular anisoplanatism. Because atmospheric phase distortions depend on the direction in the sky, the PSF varies over the field of view. Basically, the further from the centre of the image, the lower the Strehl ratio and the larger the FWHM. This means that the fraction of light contained in a given aperture is going to vary and that the brightness of the objects far from the centre will be underestimated.

To assess the consequences of anisoplanatism on accurate photometry, we used simulated point spread functions constructed using the method described in Wilson \& Jenkins (1995) and kindly provided by the authors. We simulated PSFs corresponding to different distance from the reference star $(0,5,10,15$ and 20 arcsec $)$. For each PSF, we applied the IRAF/NOAO APPHOT package to work out the flux contained in the three different apertures defined as before by the first dark rings. We performed the operations for three values of $D / r_{0}: 5,7.5$ and 10 . These correspond approximately to the $K, H$ and $J$ bands on a 4-meter telescope in good seeing conditions ( 0.75 arcsec seeing at $0.5 \mu \mathrm{m})$. The variation in magnitude due to anisoplanatism is given in Table 4 for the three bands and the four separations. All results are relative to the magnitude of an object placed at the centre of the image (the guide star).

Table 4 shows that the variations in magnitude are much higher when the size of the aperture is defined by the first dark ring, rather than the other ones. The reason
Table 4. Variation in magnitude due to anisoplanatism as a function of the distance to the reference star. Results are given in the $J, H$ and $K$ bands for a 4-meter telescope in good seeing conditions. The number of the dark ring defining the aperture is given in the second column. The variations are given relative to the magnitude of an object placed at the centre of the image. As a comparison, the angle for which the Strehl ratio is attenuated by 50 per cent compared to the on-axis value is $17.5^{\prime \prime}$ in $J, 20.8^{\prime \prime}$ in $H$ and between $24^{\prime \prime}$ and $25^{\prime \prime}$ in $K$

\begin{tabular}{cccccc}
\hline Band & Ring & $5^{\prime \prime}$ & $10^{\prime \prime}$ & $15^{\prime \prime}$ & $20^{\prime \prime}$ \\
\hline \multirow{3}{*}{$J$} & 1 & 0.035 & 0.130 & 0.25 & 0.44 \\
& 2 & 0.006 & 0.020 & 0.04 & 0.10 \\
& 3 & 0.006 & 0.023 & 0.04 & 0.08 \\
\hline \multirow{3}{*}{$H$} & 1 & 0.022 & 0.082 & 0.16 & 0.29 \\
& 2 & 0.002 & 0.015 & 0.03 & 0.06 \\
& 3 & 0.003 & 0.013 & 0.02 & 0.04 \\
\hline \multirow{3}{*}{$K$} & 1 & 0.012 & 0.045 & 0.09 & 0.16 \\
& 2 & 0.002 & 0.008 & 0.02 & 0.03 \\
& 3 & 0.001 & 0.005 & 0.01 & 0.02 \\
\hline
\end{tabular}

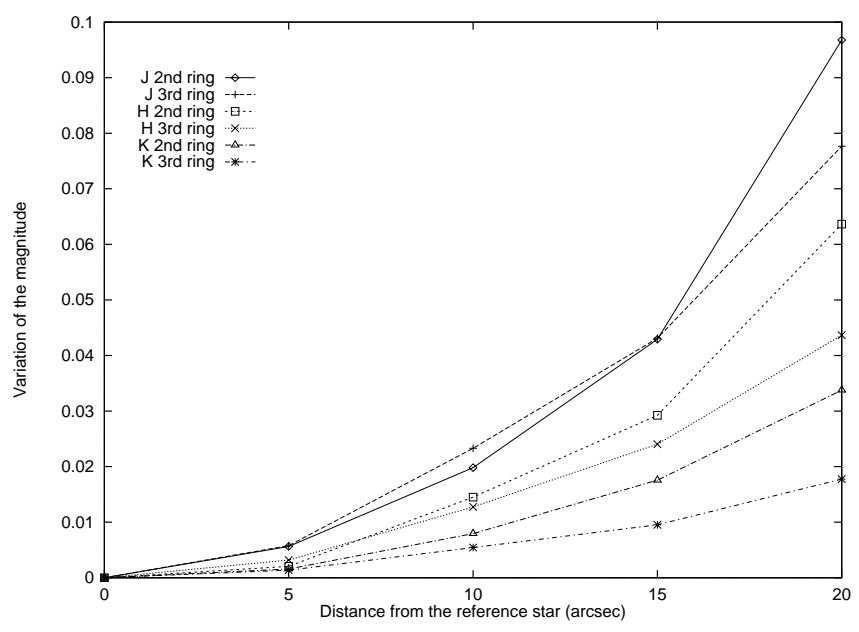

Fig. 4. The effect of anisoplanatism on aperture photometry. The error on the estimated magnitude is given as a function of the angular separation in the $J, H$ and $K$ bands and for apertures defined by the second and the third dark ring

for such a difference is easy to understand: the effects of anisoplanatism consist not only in a decrease of the Strehl ratio with the distance to the centre but also a distortion and a broadening of the core of the PSF. When an object is far from the centre, a significant part of the core spreads beyond the first dark ring, thus the large variations in the magnitude. For larger apertures, this effect has no significant consequence and the variations are much smaller. Globally, this means that the use of the smallest aperture 
should be avoided in most cases, except maybe in the $H$ and $K$ band for a small field of view.

Figure 4 presents the variations of the magnitude as a function of the angular separation for apertures defined by the second and the third dark ring. The curves for an aperture limited to the first dark ring have the same shape but at a higher level. Figure 4 allows an estimate of the variations due to anisoplanatism for apertures defined by the second and third dark ring. Of course, the variations in a real case depend on several factors like the seeing, the number and strengths of turbulent layers, the central obscuration of the telescope and other parameters of the adaptive optics system. For this reason, Fig. 4 cannot be used to compensate accurately for the effects of anisoplanatism. But it can still be used to illustrate the behaviour of the error. Basically the variations in magnitude are quite small near the centre of the image and start to increase to significant levels at a distance which depends on the band and the chosen aperture. As an example in our case, for an aperture defined by the second dark ring, the variations reach a level of 0.02 magnitude at $9 \operatorname{arcsec}$ in $J, 12 \operatorname{arcsec}$ in $H$ and $16 \operatorname{arcsec}$ in $K$.

\section{Faint structures around a star}

\subsection{Detecting structures around a star}

When trying to detect a faint structure around a star, either a companion or an extended object, the easiest solution is to subtract a point spread function, properly scaled and placed, from the image of the main star. This procedure should theoretically remove the light from the star and leave only the surrounding structures. But here again some problems appear. The adaptive optics PSF is the sum of two components: a diffraction-limited core surrounded by a large halo due to variations of uncorrected high order modes and imperfectly corrected low order modes. This halo is therefore affected by strong variations with time which are independent of the fluctuations of the central core. These variations are even higher when two different objects are compared because of the halo's dependence on parameters like the brightness of the guide star.

To assess the limits for detection of a faint companion next to a star, we used observed images of the single stars HR 2019 and HR 2076, and subtracted one from the other. For each image of HR 2019, the integration time was 75 seconds, the signal to noise ratio 7500 and the Strehl ratio 0.26 . For HR 2076, these parameters were respectively 200 seconds, 10000 and 0.22 . The delay between the two sets of images was 10 minutes. The subtraction method was applied to four different couples of images in order to obtain different residuals. Finally, we computed the standard deviation of these residuals. To work out the average detection limit for a point like object at a given distance of a bright star, we computed an azimuthally averaged radial profile of the standard deviation of the different residuals.

Figure 5 shows the radial dependence of the detection limit. The full line corresponds to the profile a star. The dotted line shows the radial profile of the standard deviation of the four different residuals. For example, we could detect on this basis a companion fainter by about 7 magnitude at 1 arcsec from the main star and fainter by more than 9 magnitudes at 2 arcsecs. This result is in excellent agreement with the observations by Tessier (1995). Note that far from the star (more than about 2 arcsec), the uncertainties are dominated by readout and sky noises. An important point is that Fig. 5 shows azimuthally averaged profiles. But the shape of the PSF is far from having a circular symmetry. Lots of non-axisymmetric small features appear, especially on the first diffraction rings. Figure 5 should therefore be considered as an over-optimistic estimate.

As an illustration of this method, we used adaptive optics data taken on January 21st 1996 to look for a possible companion to the star Betelgeuse. We subtracted the images of different calibration stars from images of Betelgeuse. No companion was visible down to the approximate limits indicated above. Near to the star, the main problem was the presence of several residual features whose position and intensity changed depending on the calibration star used. One of these features stood out by being always present at a position approximately constant. If this feature were real, it would lie at about 0.5 arcsec to the south-west from Betelgeuse and be 4.5 magnitude fainter. These data would be consistent with a reported detection by Karovska et al. (1986) using speckle observations. More details are given in Esslinger (1997).

The previous test gives us an estimate of the global noise affecting the subtraction procedure. It would be interesting to work out the part due to the each main cause of noise. With this aim in mind, we compute the standard deviation of our set of 75-second exposures of HR 2019. This gives us the detection limit if we only had to deal with variations of high order modes and photon noise, and did not have the difficulty of mismatch between PSFs from different stars. The radial dependence of this limit corresponds to the dashed line in Fig. 5. This shows that the main source of error in our procedure is indeed the variation of the PSF between the observations of the science object and the reference star, confirming the results by Tessier (1995).

We can also compare the relative contribution of photon noise compared to the noise introduced by uncorrected or imperfectly corrected modes. We can work out the photon noise from the number of photons per pixel in the original image at a given distance from the peak. We find for instance that, at the centre of the PSF, the level of photon noise is about 7 magnitudes fainter than the peak intensity, thus 3 to 4 magnitudes fainter than the dashed line in Fig. 4. At a distance of 1 arcsec, photon noise is 


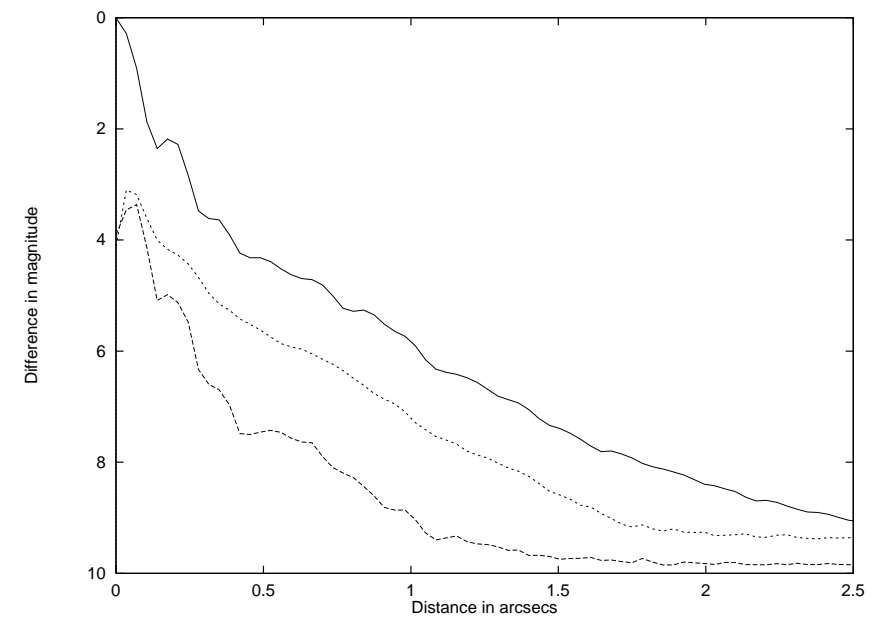

Fig. 5. Three radial profiles (in magnitude and relative to the main star). The full line is the profile of the star before subtraction. The dotted line shows the radial profile of the standard deviation of four different residuals. This gives us the limiting magnitude for detection of a companion at a given distance from the main star. The dashed line (the lowest) corresponds to the standard deviation of a set of four images taken on the same star

10 magnitudes fainter than the peak intensity and thus 1 magnitude below the same line. From this, we see that photon noise is usually not important compared to the noise introduced by uncorrected or imperfectly corrected modes.

These results are obtained in a direct mode. Obviously the search for faint structures around a bright object can be much improved by using a coronagraph. For example, Beuzit et al. (1997) used a coronagraph with the Adonis system to study the circumstellar disk around Beta Pictoris. In their observations, they would have been able to detect a companion fainter by 11 magnitude at 1 arcsec and by 13 magnitudes at 2 arcsecs. This is an improvement of 4 magnitudes compared to our results.

\subsection{Measuring structures around a star}

The subtraction procedure can be used to carry out photometric measurements. Another possibility is to use a photometric method designed for such cases: point spread function fitting, as for example in DAOPHOT (Stetson 1987). To assess the accuracy of photometry in this case, we took images of the star HD 5980 and its calibration star SAO 255763 observed in December 1995 in the $K$ band. For HD 5980, the integration time was 200 seconds, the signal to noise ratio was 7000 and the Strehl ratio 0.32 . For SAO 255763, these parameters were respectively 120 seconds, 45000 and 0.35 . The delay between the images of the two objects was 20 minutes and the pixel scale 0.050 arcsec. With the image of HD 5980, we artificially created an image of a main star and its faint companion situated at a given distance and with a given difference in magnitude. The position of the companion was chosen to avoid the diffraction spikes of the main star and to limit the influence of the other residual features. We also performed the same procedure on images of Betelgeuse and its calibration star HR 2076. The integration time for the images of Betelgeuse and HR 2076 were respectively 6 and 200 seconds, the signal to noise ratios 25000 and 10000 , and the Strehl ratio 0.22 for both objects. The delay between the two observations was 10 minutes and the pixel scale 0.035 arcsec.

We then applied the point spread function fitting algorithm DAOPHOT to these images, providing it with an image of the right calibration star. Comparing the result of DAOPHOT and the known magnitude of the companion then enabled us to work out the error in the magnitude estimation. As a comparison, we also performed the same procedure with other stars but no significant differences appeared in the results.

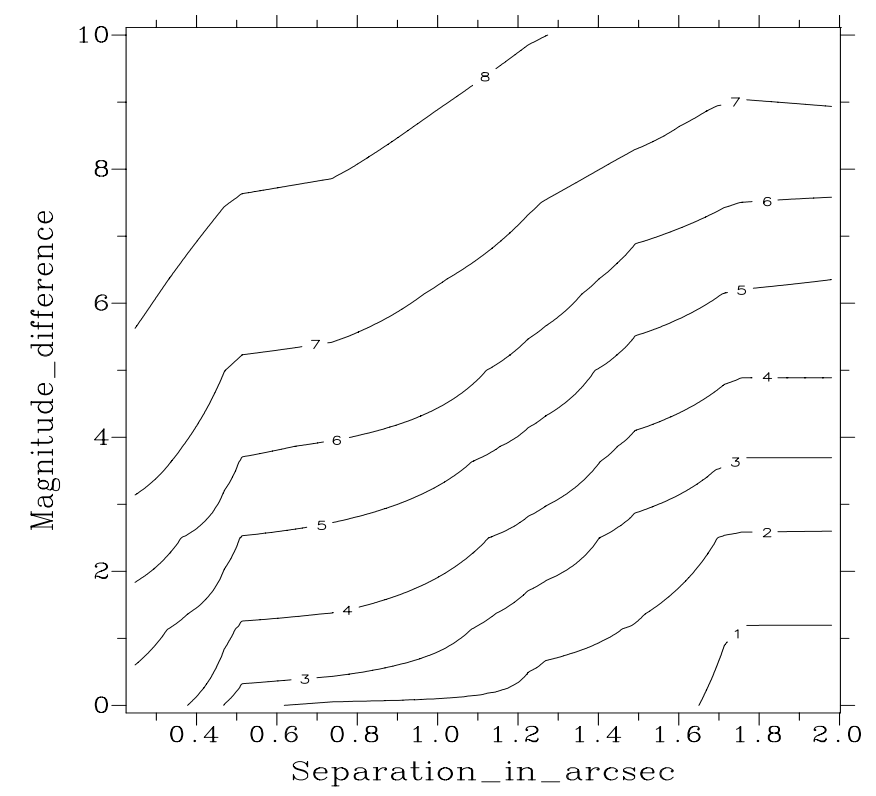

Fig. 6. Error in the photometry of a faint companion as a function of the distance and the difference in magnitude. The original image was created with an image of Betelgeuse and DAOPHOT used an image of HR 2076. The contours are 0.001 (1), 0.003 (2), 0.01 (3), 0.03 (4), 0.1 (5), 0.3 (6), 1 (7) and 3 (8) magnitudes

\subsection{Performances}

Figures 6 and 7 present results of such investigations as contour plots. They show, for given separations and differences in magnitude, the error which is made in the magnitude estimated by DAOPHOT. Figure 6 was created using an image of Betelgeuse and Fig. 7 using an image of 


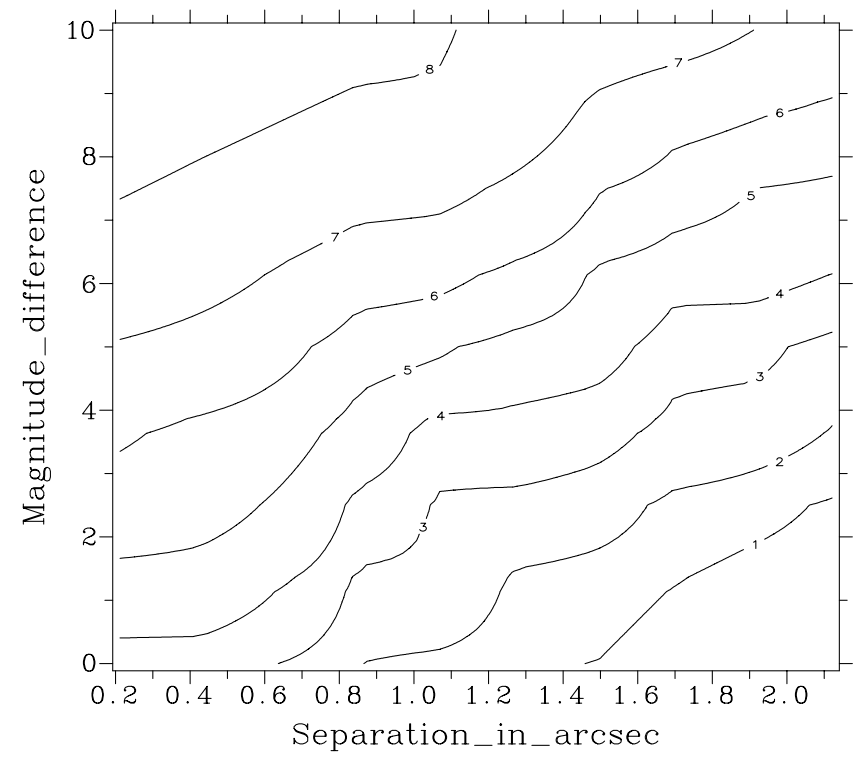

Fig. 7. Error in the photometry of a faint companion as a function of the distance and the difference in magnitude. The original image was created with an image of HD 5980 and DAOPHOT used an image of SAO 255763. The contours are 0.001 (1), 0.003 (2), 0.01 (3), 0.03 (4), 0.1 (5), 0.3 (6), 1 (7) and 3 (8) magnitudes. Note that the range in separation is not exactly the same as in the previous figure

HD 5980. For Betelgeuse, we varied the separation from 0.25 to 1.98 arcsec with a step of 0.25 arcsec. In the case of HD 5980, this was done for a separation between 0.21 and 2.12 arcsec with a step of 0.21 (the difference is mainly due to the different pixel size). In both cases, we considered differences in magnitude between 0 and 10 with a step of 1.25 magnitude. The contour plots were smoothed to minimise the small irregularities induced by residual features in the PSF.

Figure 7 can be considered representative of the performances of DAOPHOT in good conditions. It gives an estimate of the best performance an adaptive optics system can achieve when measuring the light of a faint companion next to a bright star. The figure shows that a very good photometric accuracy (an error of about 0.01) can only be achieved further than about 1 arcsec and for a difference in magnitude not larger than 3 or 4 . Good photometric accuracy (an error between 0.01 and 0.1 ) can only be achieved for a difference in magnitude less than 2 near the star, and for a difference of 6 or 7 magnitudes further than 1 arcsec. Only a poor accuracy (more than 0.1 ) is available near the main star in most cases and, beyond 1 arcsec, for differences in magnitude larger than 6 or 7 .

When comparing the two figures, it can be noted that the results are slightly better in the case of HD 5980. This is due to a longer exposure time (200 seconds instead of 6 ), therefore smaller fluctuations in the halo, and also to a more careful choice of calibration star. But note that the two figures, even though they were obtained in very different conditions and during different runs, are nevertheless very similar, which indicates that they can be considered typical and unlikely to be much improved in similar atmospheric conditions. We carried out a similar procedure using images of HD 5980 and SAO 255763 obtained in the $H$-band. The integration times were respectively 200 and 100 seconds, the Strehl ratios 0.13 and 0.15 , the signal to noise ratios 5500 and 35000 , and the delay between the images 15 minutes. Again the results were very similar, only slightly worse. For a difference in magnitude of 2.5 between the main star and its companion, the errors in the magnitude estimation were respectively $0.15,0.02$ and 0.002 at $0.5,1$ and 2 arcsec, compared to $0.15,0.015$ and 0.001 in the $\mathrm{K}$ band for the same couple of objects. For a difference in magnitude of 5 , the errors were $0.6,0.2$ and 0.02 in $H$, compared to $0.6,0.15$ and 0.01 in $K$. This result confirms that only slight improvement is obtained when the Strehl ratio is higher, at least in the range 0.10 to 0.35 . Better results could obviously be obtained for higher Strehl ratios, say above 0.4 .

\section{Crowded fields}

\subsection{Accuracy of PSF fitting}

The most usual case of a crowded field is a star cluster. The accuracy of photometry in such a situation as been assessed by several authors for HST images (see e.g. Malumuth et al. 1991 or Sodemann \& Thomsen 1997). These studies showed that the errors on the magnitude of fainter stars could become large very quickly. For instance, Sodemann \& Thomsen (1997) studied two different crowded field observed with the HST and showed that the magnitudes of most stars were overestimated and that the errors could easily reach several tenths of a magnitude for faint stars. In this section, we assess the accuracy of photometry in crowded fields in the case of adaptive optics observations. This case is linked to the previous one, but in a way that can be complex.

We created several images of random crowded fields with a uniform mean density using the IRAF/ARTDATA package. As point spread functions, we used observed images of HD 5980 taken in the $H$ and $K$ bands. The Strehl ratios were respectively 0.13 and 0.32 and the signal to noise ratios 5500 and 7000 . The integration time was 200 seconds in both cases and the pixel size 0.050 arcsec. We used the Bahcall and Soneira luminosity function provided by the package with a 10-magnitude range (Bahcall \& Soneira 1980). This function with its default parameters gives a good fit to the observed main sequence in several nearby globular cluster. To simulate different densities of stars, we changed either the total number of stars or the size of the field of view, but kept a fixed spatial scale. We were careful to keep a number of stars large enough (at 
least 50) to provide a good representation of the average behaviour.

After creation of an image, we used the IRAF/NOAO DAOPHOT package to compute the magnitude of its stars. This was done with two different point spread functions. First, the right one, which enabled us to assess the performances of the DAOPHOT algorithm itself and the problems due to the very complex shape of the PSF, especially the presence of numerous residual features around the core, but also the unavoidable numerical errors appearing during the fitting process. Then, a different PSF was used to study the problems due to the mismatch between the actual PSF and the one obtained from a calibration star (required when no suitable PSF can be found in the cluster). As a second PSF, we used an image of the reference star of HD 5980 in the same band. The integration times for these reference stars were respectively 120 and 100 seconds, the Strehl ratios 0.35 and 0.15 , and the signal to noise ratios 45000 and 35000 . The time delay between images was 15 minutes in $H$ and 20 minutes in $K$. DAOPHOT yielded magnitudes that could be compared to the real ones used in the simulation of the star field. We worked out the photometric errors for 7 different densities: $0.15,0.5,0.75,1.5,3,5$ and 15 stars per $\operatorname{arcsec}^{2}$ (it would, of course, be impossible to do photometry on uncompensated images for the higher densities). No noise was added to the artificial image as we were concentrating on the problems due to the shape of the PSF, not on the influence of other sources of noise. Note that we supposed the positions of the stars known before application of DAOPHOT as a starting point, which would for example be the case if a star detection algorithm using a deconvolved image had been applied first (see e.g. Snell 1996). We nevertheless let the algorithm try to recentre the stars afterwards.

Figures 8 and 9 present typical results obtained through this procedure. The magnitude estimated by DAOPHOT for each star is given as a function of its real magnitude, and this is compared to the theoretical line that would be obtained if the measurement were perfectly accurate. Figure 8 has been obtained with an $H$-band PSF and two different stellar densities: 1.5 and 15 stars per $\operatorname{arcsec}^{2}$. DAOPHOT was provided with the right PSF, the same as used to create the artificial cluster (an image of HD 5980). Figure 9 has been obtained with $K$-band PSFs for a density of 15 stars per $\operatorname{arcsec}^{2}$. In this case, two results are compared, the ones obtained when using the right PSF and the ones obtained when using a wrong PSF (the reference star SAO 255763 instead of HD 5980).

Consider first the case where DAOPHOT is provided with the right PSF, as illustrated in Fig. 8. The first thing to notice is the fact that the magnitudes of the brightest stars are in general accurately estimated, even if a few of them are clearly underestimated. There is nevertheless a scatter that can be considered large for many astronomical purposes. We computed the errors on the magnitudes of

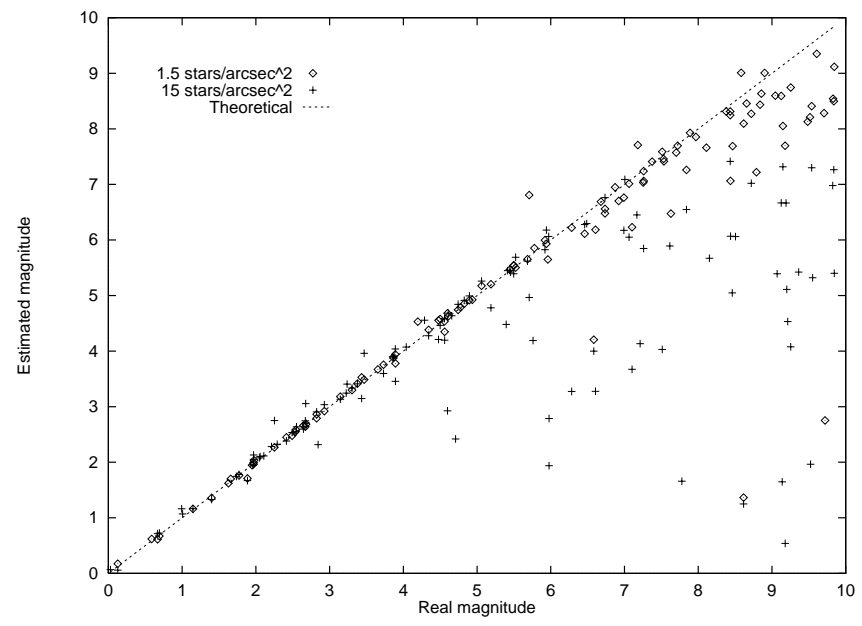

Fig. 8. Stellar magnitudes estimated by DAOPHOT in a star cluster as a function of the real magnitudes. Two densities are presented: 1.5 stars per $\operatorname{arcsec}^{2}$ and 15 stars per $\operatorname{arcsec}^{2}$. The dotted line corresponds to a perfect agreement between estimated and real magnitudes and the zero point for the magnitude scale is arbitrary. Theses results have been obtained by providing DAOPHOT with the right PSF, the same used to create the artificial cluster. The PSF was an $H$-band image of HD 5980

the brightest stars for each density and PSF. The rms error in the magnitude determination varied between 0.05 and 0.1 magnitude for densities lower than a few stars per $\operatorname{arcsec}^{2}$ and could reach 0.15 for higher densities. This was true for both $H$ and $K$ band PSFs. From this, we can already conclude that crowded fields do not allow a very good photometric precision. Even for the brightest stars, the accuracy is at best 0.05 magnitudes, which can already be a major drawback for a large number of projects.

The second feature visible in Fig. 8 is the fact that the errors increase as the star become fainter, and as a consequence the faintest stars can have their flux overestimated by several magnitudes. Of course, the lower the density, the fainter the level at which this increase of the errors occurs. Not all the faint stars are affected and the curves present a large scatter. This could be expected as the magnitude of a star is not the only important parameter in the error on its photometric estimation. Other factors are the distances to the nearest bright stars and the magnitudes of these objects.

This behaviour where stars, especially the faintest ones, have their intensity systematically overestimated can be attributed to three effects, two of which are always present in crowded fields and are not due to the use of adaptive optics. The first effect is confusion: two stars that are too close cannot be distinguished and are therefore considered by DAOPHOT as a single object, which directly leads to an overestimation of the flux. The second effect is blending: stars too faint to be detected can increase the estimated brightness of the brighter sources, 


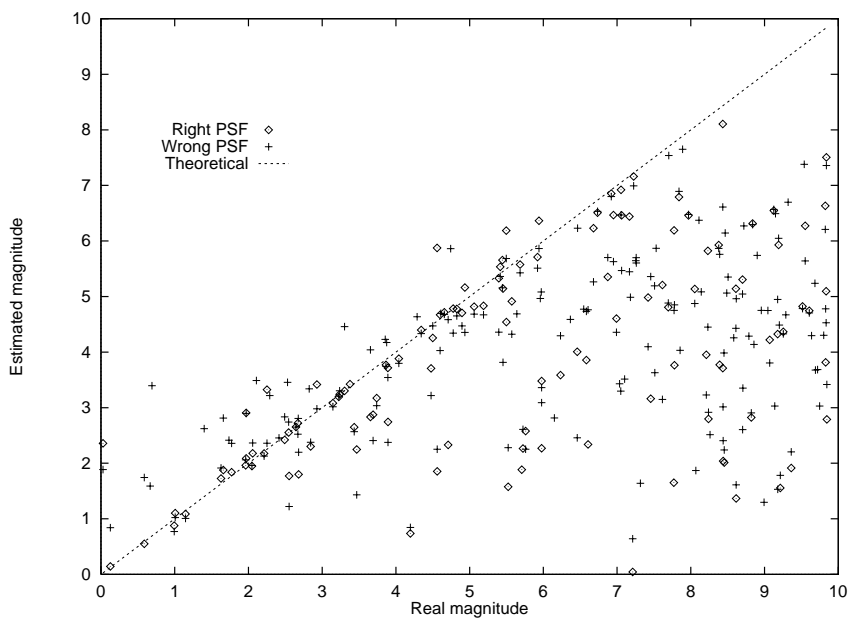

Fig. 9. Stellar magnitudes estimated by DAOPHOT in a star cluster as a function of the real magnitudes. Two cases are presented: one where DAOPHOT was provided with the right PSF (a $K$-band image of HD 5980) and one when the algorithm used a wrong PSF (a $K$-band image of SAO 255763). The density was 15 stars per $\operatorname{arcsec}^{2}$. The dotted line corresponds to a perfect agreement between estimated and real magnitudes and the zero point for the magnitude scale is arbitrary

which also lead to an overestimation. These two effects are not linked to the use of adaptive optics. On the contrary, the sharper the PSF, the less confusion there is, and the more faint sources can be detected. For this reason, adaptive optics yields a large improvement compared to normal ground-based images (see the end of this section for an illustration).

The source of uncertainty linked to the use of adaptive optics is the presence of numerous residual features in the PSF. As a consequence, some of the light contained in the residual features associated with the bright stars of the field might be redistributed into the faint stars, thus increasing their measured brightness and at the same time slightly decreasing the measured flux of the bright stars. This would be even more true when a wrong PSF is used by DAOPHOT, because of the fluctuations of these features. The importance of this effect can be estimated from Fig. 9, which compares the results in given conditions for an estimation with the right PSF and one with the wrong PSF. It can be seen that more bright sources are underestimated when DAOPHOT uses the wrong PSF, and the rms error for these sources is therefore larger. The difference is less obvious as far as fainter sources are concerned. The increase in the estimated error is then similar and no obvious difference is visible (which will be confirmed below in this section). The residual features therefore introduce some uncertainty for the brightest stars, but confusion and blending are still the major sources of noise for fainter sources. Note that the presence of large errors in the estimated magnitude of the brightest stars only occured for the highest densities, say a few stars par $\operatorname{arcsec}^{2}$.

\subsection{Influence of the stellar density}

In order to study the behaviour of photometry in crowded fields as a function of the stellar densities, we worked out for each density, the difference in magnitude relative to the brightest star at which a given level of error was reached. Given that the error are already quite significant for the brightest stars (between 0.05 and 0.15 magnitude), we chose a value of 0.2 magnitude as a threshold for the error. The measurement of the limiting difference in magnitude was carried out by viewing the previous curves with an adequate magnification. Given the very irregular distribution of points as illustrated in Fig. 8, this limiting difference is sometimes difficult to estimate precisely, and the uncertainty in it is of the order of 0.5 magnitude or a little more.

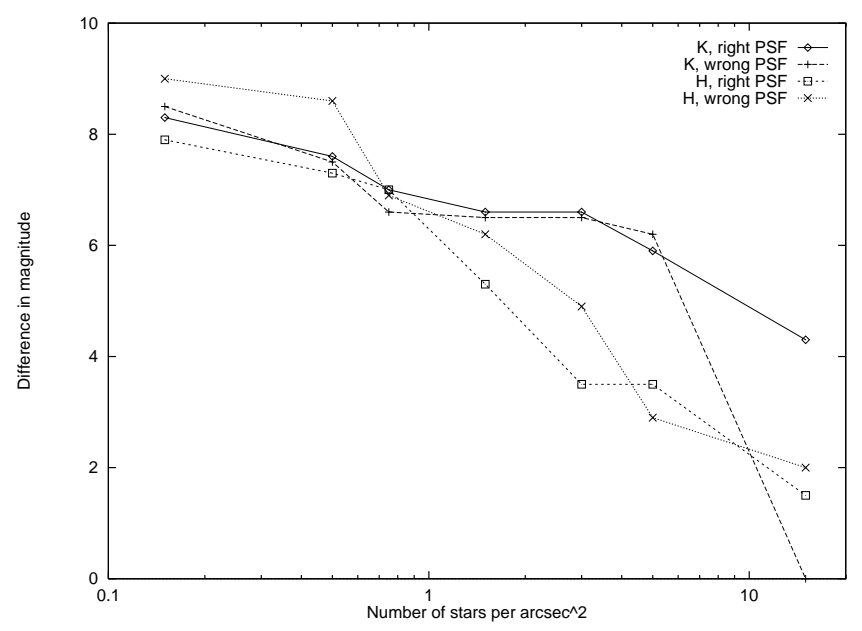

Fig. 10. The difference in magnitude relative to the brightest star at which the error on the estimated flux reaches a level of 0.2 magnitude, as a function of the stellar density. Four sets of values are shown, corresponding to PSFs obtained in the $H$ and $K$ band and to either the right or the wrong PSF for the application of DAOPHOT. The uncertainty on each point is of the order of 0.5 magnitude

Figure 10 gives the limiting magnitude defined above as a function of the stellar density. Four curves are presented, two with $H$ band PSFs, two with $K$ band PSFs, two when DAOPHOT used the right PSF and two when the algorithm used a wrong PSF. It can broadly be seen that for low densities, an error of 0.2 is reached for stars between 8 and 9 magnitudes fainter than the brightest source in the field. Note that the limiting magnitudes in this case is partially due to the limited signal to noise of the PSFs used for the creation of the artificial cluster (5 500 in $H$ and 7000 in $K$, corresponding to 9.4 and 9.6 magnitudes between the peak and the background noise). When the density increases, the limiting difference in magnitude goes down, reaching about 3 in $H$ and 6 in $K$ for a density of a few stars per $\operatorname{arcsec}^{2}$. At more than 10 stars 
per $\operatorname{arcsec}^{2}$, the limiting difference reaches 2 in $H$ and about 4 in $K$. But note that in the case of mismatched PSFs in the $K$ band, an error of 0.2 magnitude is already present for the brightest sources. This might be due to a high level of fluctuations in the residual features as explained before. Several other features can be noted. First, with the exception of the highest density, the curves obtained in a given band with the right or the wrong PSF are very similar. This confirms that the mismatch between PSF is less important than the confusion and blending problems at this level. Second, still with the exception of the highest density, the results are better in the $K$ band. This is clearly the result of a better Strehl ratio, which decreases the effects of confusion and blending.

As a conclusion, we can note that photometry in a crowded field leads to inaccurate results, with errors between 0.05 and 0.1 magnitude for the brightest stars, and reaching several tenths of a magnitude and even one magnitude for fainter stars. But adaptive optics is not directly responsible for this problem. On the contrary, the use of this technique strongly reduces confusion and blending and therefore allows a linear behaviour until fainter sources. To compare adaptive optics and normal results, we also created artificial images of a cluster using a seeinglimited PSF with a gaussian shape and a FWHM of 1.0 arcsec We chose two densities, 1 and 10 stars per arcsec ${ }^{2}$, and performed the same procedure as for the adaptive optics images, using the same PSF for the fitting algorithm and the creation of the artificial image. Figure 10 shows that adaptive optics images yield a precision better than 0.2 magnitude down to $\Delta m=6.5$ for a density of one star per $\operatorname{arcsec}^{2}$ and down to $\Delta m=2$ or 4.5 for 10 stars per $\operatorname{arcsec}^{2}$ (assuming the right PSF is used). Moreover, for the brightest stars, the errors in the estimated magnitudes are respectively 0.05 and 0.1 magnitude or so. For the seeing limited images, the measurements of the brightest stars were already wrong by about 0.5 magnitude for both densities. In the case of a density of one star per $\operatorname{arcsec}^{2}$, for stars fainter than $\Delta m=3$, some measurements were affected by errors between 1 and 2 magnitudes, and beyond $\Delta m=6$, the errors kept on increasing rapidly. For the higher density, an error of 1 magnitude already occured at $\Delta m=2$. The use of adaptive optics therefore brings a significant improvement in the photometry of crowded fields compared to seeing-limited image, but it has to be kept in mind that the error on the flux of faint sources can rapidly become very large.

\subsection{Angular anisoplanatism}

We also assessed the performances of point spread function fitting in clusters as far as angular anisoplanatism is concerned. We used the same simulated PSFs as in the study of angular anisoplanatism in aperture photometry. For each value of $D / r_{0}$ and each separation, we tried to fit the on-axis PSF to the off-axis one using DAOPHOT.
Comparing the result with a fit of the on-axis PSF to itself gave us an estimation of the error due to PSF distortions induced by angular anisoplanatism. Note that DAOPHOT needed an aperture size to make a first guess of the magnitude. We checked that the final estimate was independent of the choice of this aperture size.

Table 5. Error in the magnitude estimated by DAOPHOT in three bands and as a function of the distance to the centre in arcsec

\begin{tabular}{ccccc}
\hline Band & $5^{\prime \prime}$ & $10^{\prime \prime}$ & $15^{\prime \prime}$ & $20^{\prime \prime}$ \\
\hline$J$ & 0.07 & 0.28 & 0.53 & 0.95 \\
$H$ & 0.05 & 0.17 & 0.34 & 0.62 \\
$K$ & 0.02 & 0.09 & 0.17 & 0.32 \\
\hline
\end{tabular}

Table 5 presents the results of this study. The error in the magnitude estimation is given in the $J, H$ and $K$ bands for four different distances to the centre. Comparing Table 5 to Table 4 shows that the level of error is much higher in this case, by about a factor 2 compared to the error for aperture photometry using an aperture size defined by the first dark ring. This high level is obviously a important drawback. Even in $K$ for a distance of 5 arcsec, the error is already about 0.02 magnitude. Note that some work is in progress to enable DAOPHOT to take into account large variations of the PSF in the field of view and the situation should therefore improve (Stetson 1994). Also note in a real case, the level of variation of the PSF depends on several factors like the seeing, the number and strengths of turbulent layers or the central obscuration of the telescope. The results obtained in Table 5 should therefore only be considered as illustrative.

\section{Photometry on deconvolved images}

\subsection{Deconvolved images}

So far, we have always applied photometric methods on original images (i.e. without post-processing). But even if these carry a lot of information, the full exploitation of adaptive optics images often requires the use of deconvolution methods. It is therefore also important to study how accurate photometry using deconvolved images can be. The method in this case is to use aperture photometry on the restored image with a very small aperture. It would be awkward to use a PSF fitting algorithm as the stellar images in the result of the deconvolution are very sharp (basically each point like object has its flux contained in a single pixel) (Cohen 1991).

Several studies of the photometric accuracy of deconvolved images have been published, especially in the case of the HST before correction of spherical aberration. 
Cohen (1991) studied the accuracy in the photometry of a crowded field after deconvolution using the maximum entropy method. She showed that the algorithm recovered faint sources systematically too faint and that it could therefore not be used when accurate photometry was needed. She also introduced a standard procedure which consisted in performing deconvolution on the original image in order to generate a list of stars and applying a PSF fitting algorithm to the original image using this list. Linde \& Spännare (1993) concentrated on the Lucy-Richardson method. They showed that in a crowded field the brightness of most faint stars and some bright stars was systematically overestimated, whether using the original image or a deconvolved one. This was due to the confusion problem, where close stars were measured together as a single object. They also showed that the Lucy-Richardson method could decompose many such instances and allow a linear behaviour at slightly fainter levels of brightness. Other more recent papers on this subject include Lindler et al. (1994) and Busko (1994) which compare the results for several deconvolution techniques.

Several authors have studied the performances of different deconvolution methods in the case of adaptive optics images but concentrating on the appearance of the reconstructed image rather than photometry. Christou \& Bonaccini (1996) applied several linear and non-linear methods, including blind and short-sighted deconvolution, to observations of the double star $\mathrm{T}$ Tauri (separation about 0.7 arcsec). When trying to determine the difference in magnitude between the two components, they obtained values varying from 1.46 to 1.85 depending on the method used, a very large range of 0.4 magnitudes or so. Tessier (1997) also applied different methods to a binary stars with a separation of about 0.13 arcsec. His estimation of the difference in magnitude varied from 0.74 to 0.96 , that is to say a range of about 0.2 magnitudes. From these results, we can easily see that the problem of photometry on deconvolved images is far from being settled.

\subsection{Deconvolution with a correct PSF}

To estimate the precision of photometry on deconvolved images, we considered the detection of a faint companion next to a bright star, a case easier to analyse than star clusters. We first started with the case where the PSF was accurately known (for example if an isolated star is available in the field of view). Again, we created artificial images of stellar couples with different separations and magnitudes. We first applied DAOPHOT as a reference, then applied a deconvolution method to the image using the same PSF as for the creation of the image. Finally, we used aperture photometry with a very small aperture to perform photometry on the deconvolved image. We used the two main deconvolution algorithms currently popular in the adaptive optics community: the maximum entropy method and the Lucy-Richardson algorithm, as provided by the IRAF/STSDAS RESTORE package.

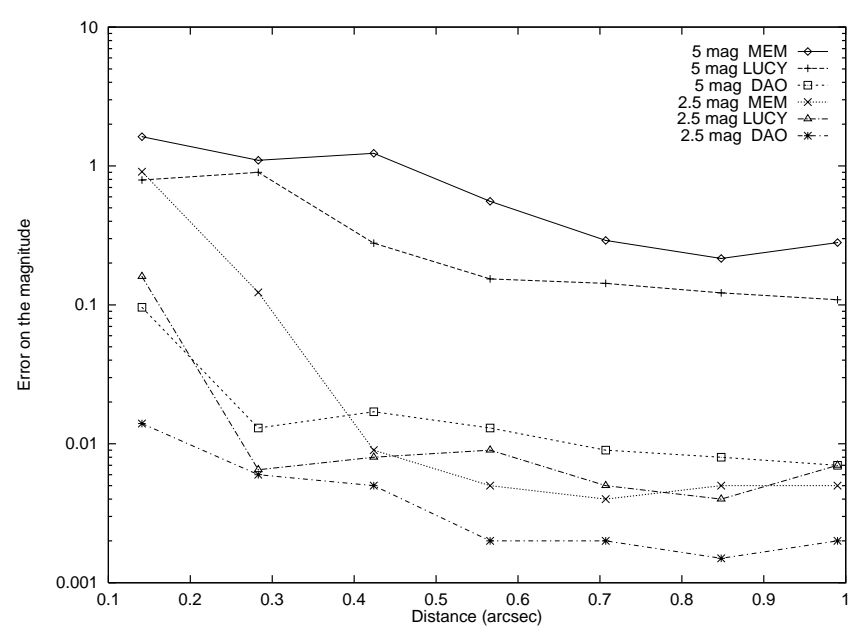

Fig. 11. Error in the magnitude of a faint companion as a function of the separation. Two differences in magnitude between the main star and its companion are considered: 2.5 and 5 . Results are given for DAOPHOT on the original image (DAO) and for aperture photometry on the image deconvolved by the maximum entropy method (MEM) and the Lucy-Richardson algorithm (LUCY). The PSF used was an image of HD 5980 and no noise was added to the original frame

Figure 11 presents the resulting error on the estimated magnitude as a function of the separation. Two differences in magnitude between the main star and its companion were considered: 2.5 and 5 . In each case, three values are given: the result from DAOPHOT applied to the original image and the results given by aperture photometry on the images deconvolved by the maximum entropy and the Lucy-Richardson methods. The PSF used was an image of HD 5980 in the $K$ Band. The star had been observed with an integration time of 200 seconds, a Strehl ratio of 0.32 and a signal to noise ratio of 7000 . We wanted to estimate the errors due specifically to the shape of the adaptive optics PSF and therefore assumed there was no noise in the original image.

Figure 11 shows that the two deconvolution methods yield larger errors than PSF fitting, as could be expected from previous studies. For a large difference in magnitude between the main star and its companion, the errors after deconvolution are more than 10 times the errors with DAOPHOT, the maximum entropy method performing a little worse than the Lucy-Richardson algorithm. When the difference in magnitude is smaller, the error after deconvolution is only a few times larger than the one with DAOPHOT at large separation, but increases drastically when the separation decreases, the maximum entropy method still performing a little worse. We checked these results by computing the error for several differences 
in magnitude (from 1 to 5 ) and for two given separations ( 0.28 and 0.85 arcsec). The previous behaviour was confirmed, with the error for photometry after deconvolution increasing from a few times the error with DAOPHOT for small differences in magnitude, to about 10 times for large differences.

\subsection{Deconvolution with a wrong PSF}

As we already noted, the PSF usually has to be worked out from the image of a calibration star observed at a different time than the science object. The variations of the PSF with time then induces errors in the deconvolution process and the subsequent photometric measurements. In order to study this problem, we applied the same procedure as before but using different PSFs for the creation of the binary star image and its deconvolution. We used the objects that provided the best result with DAOPHOT: HD 5980 and its calibration star SAO 255763. The integration time for the image of HD 5980 was 200 seconds, the signal to noise ratio 7000 and the Strehl ratio 0.32 . For SAO 255763, these parameters were respectively 120 seconds, 45000 and 0.35 . The delay between the images of the two objects was 20 minutes. We choose two different separation, 0.28 and 0.85 arcsec, and studied the error on the magnitude of the companion as a function of the difference in magnitude between the two stars. As before, we introduced no noise in the original image.

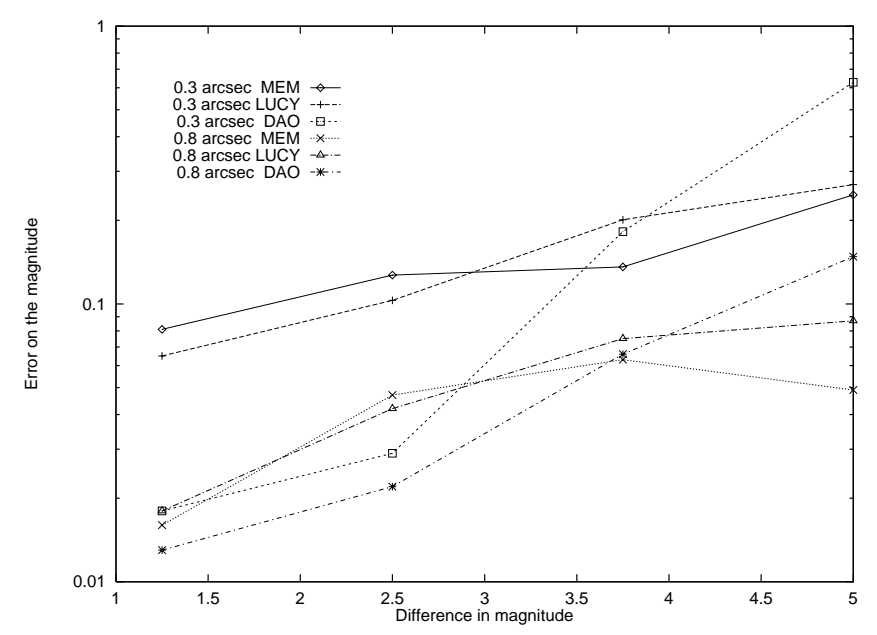

Fig. 12. Error in the magnitude of a faint companion when using a wrong PSF. The results given by DAOPHOT on the original image (DAO) and by aperture photometry applied on the images deconvolved by the maximum entropy method (MEM) and the Lucy-Richardson algorithm (LUCY) are shown. Two separations were considered: 0.28 and 0.85 arcsec (noted 0.3 and 0.8 in the figure)

The results are presented in Fig. 12. Errors obtained with DAOPHOT applied on the original image (but with a wrong PSF) and with aperture photometry on the images deconvolved by the maximum entropy method and the Lucy-Richardson algorithm are shown. As could be expected the global level of error is larger than before. For example, for a difference in magnitude of 2.5 and a separation of 0.8 arcsec, DAOPHOT and the Lucy-Richardson method yield errors of about 0.02 and 0.04 in this case, and about 0.002 and 0.004 in the previous case. At differences in magnitude of less than 4 or so, the expected behaviour is observed, with DAOPHOT performing better than the deconvolution methods. But a surprising feature appears in Fig. 12. Given the fast increase of the error with the difference in magnitude for DAOPHOT and the slow increase for the deconvolution methods, there is a domain where aperture photometry on deconvolved images produces smaller errors than DAOPHOT applied on the original images. This occurs only in the case where a badly determined PSF is used. The threshold for this behaviour is a difference in magnitude of about 3.8, a value that only varies a little with the separation.

\subsection{The influence of noise}

The presence of noise in the images, whatever its origin, is obviously an important factor in the quality of deconvolution. To illustrate the behaviour of photometry on deconvolved images in the presence of significant noise, we carried out a study similar to the previous one but added different levels of noise to the images to be deconvolved. This noise was gaussian and the same on the whole field of view. We studied only a given case: two stars with a separation of 0.85 arcsec and a difference in magnitude of 5 (this was the unusual case where deconvolution yielded better results than DAOPHOT previously). As before, the deconvolution used a wrong PSF. The PSFs used were the same as in the previous paragraph, the only difference being the addition of noise.

Figure 13 presents the result of this study. The error in the magnitude of the faint companion is given as a function of the signal to noise ratio of its peak intensity. Three methods are compared: DAOPHOT on the original image and aperture photometry on images deconvolved by the maximum entropy method and the Lucy-Richardson algorithm. The error obtained with DAOPHOT is basically constant provided the signal to noise ratio is higher than 3. The errors using deconvolved images are much more dependent on the noise. They are stable for a signal to noise higher than 20 or 50 depending on the method, but increase drastically below these thresholds, reaching more than 1 magnitude for a signal to noise lower than 5 or so. Note that the unusual case where deconvolved images yield better results than DAOPHOT on original images is therefore limited to good signal-to-noise ratios. 
Table 6. The capabilities of photometry with adaptive optics for three different kinds of accuracy requirements. These results assume that sources of noise not linked with the use of adaptive optics are negligible. The correction is supposed to be fair (Strehl ratios between 0.15 and 0.3). Angular anisoplanatism effects assume a 4-meter telescope in good seeing conditions. Results for the detection of a companion, with or without deconvolution, apply to the $K$ band, they also assume no artifacts near the objects studied. Deconvolution always requires a high signal-to-noise ratio for the companion's peak intensity, say between 10 and 50 depending on the object and the deconvolution method. FOV stands for field of view

\begin{tabular}{|c|c|c|c|}
\hline Required accuracy & $0.1-0.2 \mathrm{mag}$ & $0.05 \mathrm{mag}$ & $0.01-0.02 \mathrm{mag}$ \\
\hline Uncrowded field & $\begin{array}{c}\text { No problem } \\
\text { (except for very } \\
\text { short exposures) }\end{array}$ & $\begin{array}{l}\text { Problems if exposure } \\
\text { time smaller than } \\
\text { a few seconds }\end{array}$ & Might be impossible \\
\hline Anisoplanatism & $\begin{array}{l}\text { No problem } \\
\text { (aperture defined by } \\
\text { second dark ring) }\end{array}$ & $\begin{array}{l}\text { FOV limited to } \\
15^{\prime \prime} \text { in } J, 17^{\prime \prime} \text { in } H \\
\text { and } 20^{\prime \prime} \text { in } K\end{array}$ & $\begin{array}{l}\text { FOV limited to } \\
10^{\prime \prime} \text { in } J, 12^{\prime \prime} \text { in } H \\
\text { and } 15^{\prime \prime} \text { in } K\end{array}$ \\
\hline $\begin{array}{l}\text { Flux of a faint } \\
\text { companion }\end{array}$ & $\begin{array}{c}\text { Down to } \\
\Delta m=4.5 \text { at } 1^{\prime \prime} \\
\Delta m=8 \text { at } 2^{\prime \prime}\end{array}$ & $\begin{array}{c}\text { Down to } \\
\Delta m=3 \text { at } 1^{\prime \prime} \\
\Delta m=6 \text { at } 2^{\prime \prime}\end{array}$ & $\begin{array}{c}\text { Only possible } \\
\text { beyond } 2^{\prime \prime} \text { and } \\
\text { down to } \Delta m=2\end{array}$ \\
\hline Crowded field & $\begin{array}{c}\text { Down to between } \\
\Delta m=2 \text { and } \Delta m=8 \\
\text { typically }\end{array}$ & $\begin{array}{c}\text { Impossible } \\
\text { except in } \\
\text { favourable cases }\end{array}$ & Impossible \\
\hline Anisoplanatism & $\begin{array}{l}\text { FOV limited to } \\
6^{\prime \prime} \text { in } J, 8^{\prime \prime} \text { in } H \\
\text { and } 10^{\prime \prime} \text { in } K\end{array}$ & $\begin{array}{l}\text { FOV limited to } \\
3^{\prime \prime} \text { in } J, 5^{\prime \prime} \text { in } H \\
\text { and } 7^{\prime \prime} \text { in } K\end{array}$ & Very limited FOV \\
\hline $\begin{array}{l}\text { Flux of a faint } \\
\text { companion after } \\
\text { deconvolution }\end{array}$ & $\begin{array}{l}\text { Down to } \Delta m=5 \\
\text { further than } 0.5^{\prime \prime}\end{array}$ & $\begin{array}{l}\text { Down to } \Delta m=2.5 \\
\text { further than } 0.3^{\prime \prime}\end{array}$ & $\begin{array}{l}\text { Down to } \Delta m=2.5 \\
\text { further than } 0.4^{\prime \prime} \\
\text { Accurate PSF required }\end{array}$ \\
\hline
\end{tabular}

\subsection{Artifacts}

Another important issue is the presence of artifacts in the deconvolved images, mainly due to the presence in the PSF of diffraction spikes, lumps in the diffraction rings and features created by residual aberrations. Of course the structure of the artifacts in the image depends on the adaptive optics system used, and the results presented in this section should therefore only be considered an illustration of the problem. We deconvolved an image of the single star HR 2019 with another image of the same star taken after a 3-minute delay. The observation was carried out in a narrow band at $2.08 \mu \mathrm{m}$. The two images of HR 2019 were obtained with an integration time of 150 seconds, their signal to noise ratios were about 7500 and their Strehl ratios 0.26 or so. We used the Lucy-Richardson method and allowed 30 iterations. Figure 14 presents the result of the deconvolution.

In this particular case, artifacts due to the first diffraction ring are clearly visible around the centre. They are between 4 and 8 magnitudes fainter than the central peak at a distance of about 0.15 arcsec. Artifacts due to the second ring are at 0.3 arcsec or so and 6 magnitudes fainter.
The other artifacts have magnitudes ranging from 6 to 8 relative to the central peak or even weaker. Performing the deconvolution with different numbers of iterations showed that the artifacts appeared very early after only a few iterations.

We also performed the same procedure on different couples of calibration stars. This showed that the result of a deconvolution was completely unpredictable. Sometimes only one or two artifacts due to the first ring appeared. Often there were about 10 artifacts as before. And in some cases several tens of artifacts were showing up. No known parameter such as the exposure time or the delay between the images seemed to control the number of artifacts. The conclusion of this attempts is clearly that the result of a deconvolution on adaptive optics data should not be trusted at faint levels, say for a difference of more than 3 magnitudes relative to the main star near the first diffraction ring and more than 5 magnitudes further out. Our experience suggests that repeating the procedure with several calibration stars helps, as some artifacts may disappear, but is usually not enough to remove the 


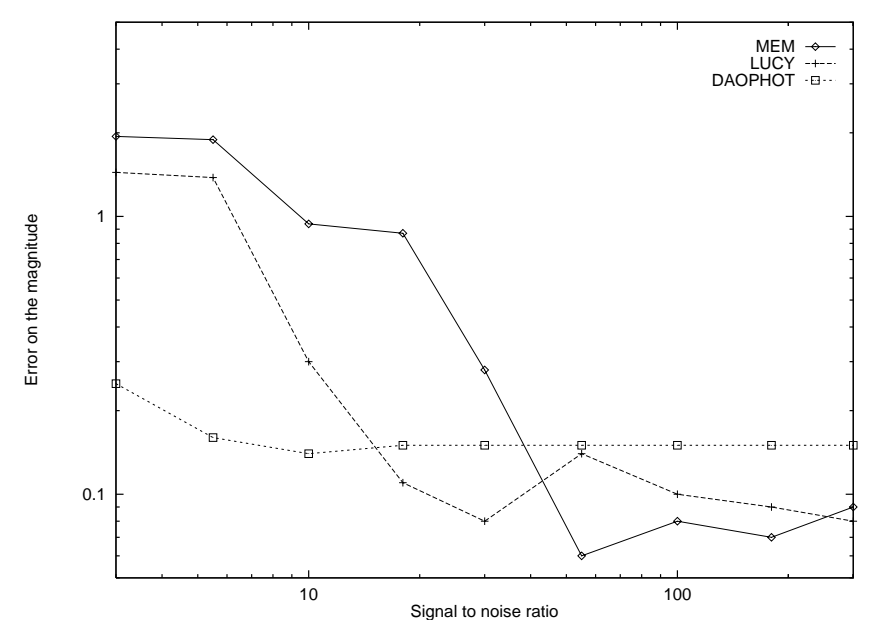

Fig. 13. Error in the magnitude of a faint companion as a function of the signal to noise ratio of its peak intensity. A wrong PSF was used and noise added into the images. The results given by DAOPHOT on the original image (DAOPHOT) and by aperture photometry applied on the images deconvolved by the maximum entropy method (MEM) and the Lucy-Richardson algorithm (LUCY) are shown. A separation of 0.85 arcsec and a difference of 5 magnitudes was considered

Fig. 14. Example of a deconvolution using a wrong PSF. An image of the single star HR 2019 was deconvolved by an image of the same star taken 3 minutes later. The exposure time is 150 seconds and the image scale 0.035 arcsec per pixel. 30 iterations were used for the deconvolution. A logarithmic scale is used for representation. In this case, artifacts due to the first diffraction ring are between 4 and 8 magnitudes fainter than the central peak. Artifacts due to the second ring are 6 magnitudes fainter. The others further out are between 6 and 8 magnitudes fainter problem completely because the main ones tend always to be present.

\section{Discussion and conclusion}

\subsection{Feasibility of astronomical programmes}

In order to summarise the results of this study, we can consider three different types of requirements for the accuracy of photometry. First, not all photometric investigations require an accuracy higher than about 0.1 or 0.2 magnitude. For instance, Longmore et al. (1990) present a way of determining the distance to a globular cluster by magnitude measurement of RR Lyrae stars in the $K$ band, where such uncertainties are acceptable. Other astronomical studies require a precision of 0.05 magnitudes or so. For example, the $H-K$ colour of a star worked out with such a precision allows the determination of its spectral type (see Koorneef 1983). Last, some astronomical projects require uncertainties to be about 0.01 or 0.02 magnitude. This is for example the case when the age and metallicity of a globular cluster has to be inferred from its near-infrared colours (see Worthey 1994, for theoretical colours of simple stellar populations). We present the results of our study in Table 6 for these three types of required precision.

\subsection{Discussion}

All the results presented so far assume that only one measurement is carried out. Performing several measurements may improve the final accuracy. Repetition can reduce the errors due to seeing fluctuations, provided these are really random and that no systematic effect appears. In order to decrease the inaccuracies due to the mismatch between the point spread functions for the object studied and its calibrations stars, lots of suitable stars have to be found, which might be a major drawback. Our experience also suggests that performing several measurements will not affect most residual features and artifacts. Moreover, in most cases it will not allow a reduction of angular anisoplanatism effects. Finally, the quantitative accuracy of deconvolved images will probably not be increased because the effects are systematic rather than random. This shows that although performing several measurements will decrease errors linked to seeing fluctuations, this method will not be able to reduce the other sources of error. So, the only revision to Table 6 by repetition of observations will be in the case of an uncrowded field and maybe in the measurement of a faint companion.

Another possible way of improving the photometric accuracy might be to use a laser guide star rather then a natural one, but the improvement is likely to be very small. Using a laser guide star does not prevent seeing fluctuations from inducing global variations in the PSF shape. The halo of the PSF is going to be fainter as more modes can be corrected, and the problem of mismatched PSFs 
will disappear, but the halo will still fluctuate with time. Residual features and artifacts will not disappear. Neither will angular anisoplanatism, though its effects can be reduced since the guide star can be moved across the object studied. Finally, the accuracy of photometry on deconvolved images has no reason to improve. All these arguments show that the results presented in Table 6 apply in the case of a laser guide star adaptive optics system, with possible improvements in anisoplanatism problems and in the measurement of a faint companion. Multiple laser guide stars (solving the cone effect) and possibly turbulence layer multi-conjugate adaptive optics systems, giving very good Strehl ratios on large fields of view, may eventually alleviate some of the problems of obtaining good photometric accuracy.

Acknowledgements. The authors wish to thank M. HeydariMalayeri and J.-L. Beuzit for providing them with data obtained during several runs on the Adonis system, and R. Wilson for his simulated point spread functions. They are grateful to H. Geoffray, P. Prado and E. Prieto for their assistance during the observations and to P. Léna for encouragement. They also thank the referee for helpful advice on improving the presentation of this work. O. Esslinger is supported by a PPARC PhD grant through the United Kingdom Adaptive Optics Programme.

\section{References}

Bahcall J.N., Soneira R.M., 1980, ApJS 44, 73

Beuzit J.-L., et al., 1994, in Adaptive Optics in Astronomy, Ealey M.A. \& Merkle F. (eds.) SPIE Proceedings Vol. 2201, p. 955

Beuzit J.-L., Mouillet D., Lagrange A.-M., Paufique J., 1997, A\&AS 125, 175

Busko I.C., 1994, in The Restoration of HST Images and Spectra II, Hanisch R.J. \& White R.L. (eds.) Space Telescope Science Institute, p. 279

Christou J.C., Bonaccini D., 1996, in Technical Report ESO
VLT Doc. No. GEN-TRE-ESO-11620-1263

Cohen J.G., 1991, AJ 101, 734

Esslinger O., 1997, PhD thesis, University of Wales, Cardiff

Gull S.F., Daniell G.J., 1978, Nat 272, 686

Heydari-Malayeri M., Rauw G., Esslinger O., Beuzit J.L., 1997a, A\&A 322, 554

Heydari-Malayeri M., Courbin F., Rauw G., Esslinger O., Magain P., 1997b, A\&A 326, 143

Karovska M., Nisenson P., Noyes R., 1986, ApJ 308, 260

Koornneef K, 1983, A\&A 128, 84

Linde P., Spännare S., 1993, in Proceedings of the 5th ESO/ST-ECF Data Analysis Workshop, Grosbol P.J. \& de Ruijsscher R.C.E (eds.)

Lindler D., et al., 1994, in The Restoration of HST Images and Spectra II, Hanisch R.J. \& White R.L. (eds.) Space Telescope Science Institute, p. 286

Longmore A.J., Dixon R., Skillen I., Jameson R.F., Fernley J.A., 1990, MNRAS 247, 684

Lucy L.B., 1974, AJ 79, 745

Malumuth E.M., Neill J.D., Lindler D.J., Heap S.R., 1991, in The First Year of HST Observations, Kinney A.L. \& Blades J.C. (eds.) Space Telescope Science Institute, p. 249

Richardson W.H., 1972, J. Opt. Soc. Am. 62, 55

Snel R., 1996, in Proceedings of the SSAB Symposium on Image Analysis, Linde P. \& Sparr G. (eds.)

Sodemann M., Thomsen B., 1997, A\&A (submitted)

Stetson P.B., 1987, PASP 99, 191

Stetson P.B., 1994, in Astronomy with the CFHT Adaptive Optics Bonnette, Arsenault R. (ed.) Proceedings CFHT Workshop, p. 72

Tessier E., 1995, in Adaptive Optical Systems and Applications, Tyson R.K. \& Fugate R.Q. (eds.) SPIE Proceedings Vol. 2534, p. 178

Tessier E., 1997, A\&AS 125, 581

Veran J.-P., et al., 1997, J. Opt. Soc. Am. A 14, No. 11

White R.L., Burrows C.J., 1990, in The Restoration of HST Images and Spectra, White R.L. \& Allen R.J. (eds.) Space Telescope Science Institute, p. 2

Wilson R.W., Jenkins C.R., 1996, MNRAS 278, 39

Worthey G., 1994, ApJS 95, 107 